http://dx.doi.org/10.1016/j.phytochem.2012.10.017

Phytochemistry

Volume 86, February 2013, Pages 127-136

\title{
Filamentous fungi from Plantago lanceolata L. leaves: Contribution to the pattern and stability of bioactive metabolites
}

Sándor Gonda ${ }^{\mathrm{a}}{ }^{*}$, Attila Kiss $^{\mathrm{b}}$, Tamás Emri ${ }^{\mathrm{c}}$, Gyula Batta ${ }^{\mathrm{b}}$, Gábor Vasas ${ }^{\text {a }}$

${ }^{\text {a }}$ University of Debrecen, Department of Botany, Division of Pharmacognosy; H-4010 Debrecen, Egyetem tér 1, Hungary;

${ }^{\mathbf{b}}$ University of Debrecen, Department of Organic Chemistry; H-4010 Debrecen, Egyetem tér 1, Hungary.

${ }^{\mathbf{c}}$ University of Debrecen, Department of Microbial Biotechnology and Cell Biology; H-4010 Debrecen, Egyetem tér 1, Hungary.

*: To whom correspondence should be addressed: Sándor Gonda: Telephone: +36-52-512-900 / 62634. Fax: +36-52-512-

943. E-mail: gondasandor@gmail.com, gonda.sandor@science.unideb.hu and Gábor Vasas, E-mail:

vasasg@tigris.unideb.hu 


\section{Abstract}

The aim of this study was to test contribution of plant-associated microorganism (PAMs) to metabolite stability/instability in a medicinal plant matrix.

Therefore, PAM strains were isolated and identified based on relevant DNA sequences from Plantago lanceolata leaves. Sterile water extracts of $P$. lanceolata were incubated with the isolated strains and antioxidants (ascorbic acid (AA), and EDTA) for 15 days, and changes in the concentrations of chief bioactive constituents (aucubin, catalpol, acteoside (= verbascoside)) were quantified by capillary electrophoresis. Phenolic breakdown-products were identified by GC-MS.

PAMs were identified from the genera Epicoccum, Bipolaris, Cladosporium, Leptosphaerulina, Aspergillus, Eurotium and Penicillium (pathongens, endophytes, and other species). Some fungi caused significant decomposition of the chief constituents $(p<0.001)$. Surprisingly, some strains inhibited breakdown of acteoside $(p<0.001)$. Meanwhile, concentration of several phenolic acids increased in fungi-infested extracts $(p<0.001)$. Gentisic acid, 4-hydroxyphenyl acetic acid, 4-hydroxybenzoic acid and hydroxytyrosol were only present when the extract was infested with a PAM. The products are powerful antioxidants and chelators. Concentrations of phenolic acids influenced acteoside stability significantly $(p<0.01)$, as shown by basic data-mining techniques. AA and EDTA also significantly inhibited acteoside breakdown in sterile model solutions $(p<0.05)$.

Our results suggest, that the phenolic acid mixture (produced during the fungal proliferation) protected acteoside from breakdown, possibly via its antioxidant activity and metal complexing ability. It was shown, that PAMs can increase or decrease the stability of chief metabolites in herbal matrices, and can significantly alter the chemical pattern of the plant matrix. 


\section{Keywords}

Plantago lanceolata L., ribwort plantain, endophyte, plant-associated microorganism, metabolite stability, phenolic acids, acteoside 


\section{Introduction}

Despite the lack of precise statistical data, it can be stated that the consumption of medicinal plants, and herbal food supplements is rising (Egan et al., 2011). Besides their widely recognized popularity, this is also due to the increasing body of evidence of pharmacological, chemical studies and clinical trials, that are accomplished to prove the effectiveness of these preparations, and aid standardization of the products. Also, there is a growing demand for evidence-based, rational phytotherapy among the scientific society and the consumers (Mukherjee et al., 2008).

The complex effects on health are caused by multiple interactions between the receptors/enzymes of the treated organism and the complex mixture of the bioactive metabolites in these natural products. A huge variability of the metabolites is typical of these matrices, which makes complete characterization very expensive - if it is possible at all. It not only makes analytical technique development more difficult, it also makes estimation of product shelf-life a task harder to solve, especially because metabolites can influence the stability of each other (Eder and Mehnert, 1998). Probably that's why papers dealing with direct stability studies of complex matrices are rare, but stability studies should be inherent parts of evidence-based phytotherapy.

The complexity is not only of chemical nature, however. Dried plant materials also contain many microorganisms that can contribute to the chemical pattern of the medicinal plant matrices by secreting their metabolites. Of course, they are only active when there is sufficient water available for their proliferation, and for their enzymes to operate. Upon re-hydration, these microorganisms use the proteins and carbohydrates of the plant material to grow, and have to detoxify some of the plants' metabolites to survive, as several metabolites have some sort of generic antimicrobial activity, as described for example for iridoid glycosides (IGs) present in Plantago lanceolata - via protein crosslinking activity (Senatore et al., 2007). Many publications are available in the literature on the 
microbiological community of medicinal plant raw materials (for a review, see (Kneifel et al., 2002)). The health hazard brought on by fungal contamination is frequently cited (Oyero and Oyefolu, 2009), many studies can be found dealing with fungal toxin contents in medicinal plants, and association of spores with some allergic diseases also appeared in the literature (Roussel et al., 2005). However, the study of effects of the plant-associated microorganisms (PAMs) on the medicinal plant bioactive metabolites and are almost completely missing.

The metabolite variability of the medicinal plants was until recently thought to be of plant origin, the contribution of microbiological agents was much less investigated. However, several metabolites were shown to be products synthetized and secreted by PAMs, or by the so-called endophytes, which organisms are very promising sources of novel pharmaceutical molecules (Gutierrez et al., 2012). The intensity of the research in this field is rising.

The selected plant for our studies is a chemically well-characterized and frequently used plant species, Plantago lanceolata L. (ribwort plantain), containing compounds different biosynthetic classes, namely, iridoid glycosides (IGs, mainly aucubin, catalpol), caffeoyl phenylethanoid glycosides (CPGs, mainly acteoside) (Fig.1.), flavonoids and polysaccharides (Ronsted et al., 2003). The former two classes are much more abundant, and are associated with interesting possible pharmacological applications. This medicinal plant and its relatives are mostly used as infusions (hot water extracts), products made of $P$. lanceolata are relatively popular in Europe. The plant is described as a welltolerated plant material commonly used to treat upper respiratory symptoms. As acteoside has a wide range of pharmacological activities (He et al., 2011), it can contribute to general health as well.

Interactions of the plants' bioactive metabolite matrix and the inherent fungal species are not well studied. The fungi may secrete bioactive metabolites into the plant matrix, but may also transform the main plant metabolites of pharmaceutical interest, so, when activated, they can influence the stability of the bioactive metabolites and change the therapeutic value of the plant material, this is 
another neglected field of phytochemical analysis.

The aim of the current work was to assess the contribution of plant-associated fungi to metabolite instability and chemical pattern in a dried plant drug model, Plantago lanceolata L. leaves. To accomplish this, we set the following goals: Isolate and identify ten fungal strains from the leaves. Quantify the degradation rates of three bioactive metabolites (aucubin, catalpol and acteoside) in the presence/absence of different fungal strains, in sterile model solutions. Identify degradation products linked to the presence of fungal activity. Run appropriate experiments to explain to observed phenomena.

\section{Results and discussion}

2.1. Isolation and identification of the fungal strains. Several strains of fungi were isolated from the plant material and their identification was accomplished using macroscopic, microscopic characteristics and by sequencing taxonomically useful regions of the purified DNA of the isolated strains. Ten well-growing strains with different visual appearance were selected for further studies. The list of identified species, and sequenced genes can be seen in Table 1. The identified ten strains were the following: 1, Aspergillus niger; 2, Eurotium repens; 3, Leptosphaerulina chartatum; 4, Aspergillus nidulans; 5, Eurotium amstelodami; 6, Cladosporium pseudocladosporioides; 7, Penicillium chrysogenum; 8, Bipolaris tetramera; 9, Epicoccum nigrum; 10, Eurotium repens. The strains are referred to as bold italic numbers (shown above) or species names throughout the manuscript. For most species, more than one DNA fragments were sequenced. The found sequences were subjected to a search in the BlastN database for identification. DNA sequences were submitted to NCBI BankIt, accession numbers are given in Table 1. Differences between our sequences and reference sequences in the database were always below $1 \%$, but full identity with database sequences was found in most cases. 
The species identified were shown to belong to many genera, and are associated with different types of plant-fungus interaction as described in the literature. Some species are described as typical endophytes, isolated several times from plant tissues. These include Epicoccum nigrum (9), which was shown to be hosted by several plant species (Wang et al., 2010) and Cladosporium pseudocladosporioides (6) could also be isolated from plant tissues, the genus contains common cosmopolitan fungi (Bensch et al., 2010). Plant pathogens were also found. A form of Leptosphaerulina chartarum (3) (Toth et al., 2007) and a form of Bipolaris tetramera (8) (Meddah et al., 2007) were described to be plant pathogens.

Other species were also found to have endophytic appearance in the literature, but should rather be considered colonizers of the plant surface, as their presence is too common. These include Aspergillus niger (1) and A. nidulans (4), that were described to have endophytic strains (Qiu et al., 2010; Liu et al., 2011), and Penicillium chrysogenum (7) was also found as an endophytic fungus of both higher plants and red sea algae (Lin et al., 2008). The members of the genus Eurotium $(2,5,10)$ were shown to colonize dried herbal material (Janda-Ulfig et al., 2009).

Presence of these strains in the herbal material have different impact of the quality of the herbal material. While some species are known to be toxin producers, there is no information on several ones regarding health risks.

\subsection{Effects of fungi on metabolite stability in P. lanceolata sterile water extracts. To}

determine the contribution of the ten fungal strains to the metabolite degradation in the plant matrix, water extracts of Plantago lanceolata were infested with fungal strains purified from the plant material and incubated for 15 days.

To test the effect of possible residual enzymatic activity coming from the plant matrix, the heatinactivated and "raw" water extract solutions were compared for bioactive metabolite stability (both 
were incubated sterile). In the changes of the metabolite levels, no significant difference could be detected $(p>0.05)$. Therefore, the effects of residual enzymatic activity was not likely to contribute to metabolite concentration changes.

Almost all of the fungi could utilize the plant extract as a medium. By the third day, strains $\mathbf{1 , 4}$, 6 and 9 developed visible colonies in the model solution. By day six, all strains except 3 developed visible mycelia on in the model solutions, and by day 9 , most of the strains produced spores as well. During development of some fungi, the change in the color of the medium was clearly visible (greenish in the case of $\mathbf{1}$, and fluorescent yellow in the case of 9). Strain 3 could not visibly grow in the water extract likely because it was sensitive to the high amount of potentially antifungal metabolites (aucubin, acteoside) present (Senatore et al., 2007).

Samples from the incubated water extracts were subjected to a capillary electrophoretic assay to quantify the concentration changes of chief constituents (aucubin, catalpol and acteoside) during the incubation time (15 days). The results are plotted in Fig. 2. The members of the fungal community decomposed the main metabolites with variable efficacy, which means that composition of the fungal community is an important factor of metabolite stability. IGs were stable in controls during the incubation period (no significant degradation detected, $p>0.05$ ), but acteoside showed an exponential decay curve, $31.5 \%$ of the initial concentration remained by the 15 th day. The compound is considered somewhat unstable in solution, as described by (Vertuani et al., 2011).

Strains 6, 8 and 9 (Fig. 2. f, $\mathbf{h}$ and i) caused a significant decrease in acteoside content of the water extract $(p<0.05)$, while decomposing IGs with different speed. Aucubin and catalpol were not equally susceptible for fungal degradation, as seen in the case of 8 and 9 (Fig. 2. h, i). A. niger decomposed IGs only ( $p<0.001)$, some strains, on the other hand, did not alter the level of IGs significantly $(p>0.05,10$, Fig. 2. b.). Different metabolites were also affected. Strain 3 did not produce any significant difference from control ( $p>0.05$, not plotted), supporting the theory that it 
couldn't proliferate in the water extract.

It was unexpected, but activity of some species caused a significant inhibition of the degradation of acteoside, examples include the strains which belong to the Eurotium species, Penicillium chrysogenum and Aspergillus nidulans (Fig. 2. b, c, d, e, g). In these cases, the residual acteoside concentration was significantly higher than in controls $(p<0.05)$, in the case of strain 4 , residual amount was $88.4 \pm 14.6 \%(p<0.001)$.

Some selected electropherograms of the incubated water extract are plotted in Fig. 3. As it can be seen, some species (e.g. 2) virtually did not alter the pattern of metabolites in the plant matrix, while others decomposed all metabolites except the IGs (e.g. 9). In the case of 2, the acteoside protection effect can also be observed.

It is important to note, that species not having significant health impact to the consumer also influenced the quality of the herbal material. Therefore, vulnerability of plant matrices for nontherapeutical purposes must be taken into account when dealing with herbal raw materials. Also, a plant pathogen (see Bipolaris tetramera, Fig. 2. h.) was shown to have several impact on stability of medicinal plant bioactive metabolites. These results reveal a new important advantage of plants coming from agricultural production over those gathered from wild populations, i.e. reduction of pathogene count by proper use of fungicides and proper post-harvest handling is likely to increase the chemical stability of the dried plant drugs. On the other hand, it was shown, that some fungal species may have beneficial effects on metabolite stability. The background of this phenomenon is dealt with later in the manuscript.

\subsection{Identification and quantification of phenolic compounds. To identify possible} breakdown-products, a GC-MS assay was carried out from the ethyl-acetate fractions of the samples after the 9th day of incubation (and a 0th day control), after derivatization. GC-MS total ion 
chromatograms (TICs) showed large differences between fungi-infested solutions and control solutions for a variety of peaks (see Fig. 4.). Metabolites were identified with the aid of the NIST Mass Spectrum Database version 2.0d library, as trimethyl-silyl (TMS) derivatives. The structures of the detected phenolic compounds are plotted in Fig. 5.

The concentration of several metabolites rose upon treatment with fungal strains. The quantitative effects of PAMs on the phenolic acid content is plotted in Fig. 6. There were differences among the strains in the pattern produced, but the activity of the nine different species led to quite similar phenolic compound patterns. Of the eight metabolites examined, untreated plant material contained caffeic acid (CA), vanillic acid (VA) and protocatechuic acid (PCA), the other molecules were present below detection limit only in controls. That is, the presence of several metabolites could be directly linked to the presence of fungal activity, these include gentisic acid (GA), hydroxytyrosol (HT), caffeic acid isomer (CAi), 4-hydroxy-benzoic acid (HBA) and 4-hydroxy-phenylacetic acid (HPAA); ( $p<0.001)$ - see Fig. 6. b., c., d., f., g. (respectively). Appearance of some of the phenolic acids was observed to be general upon fungal infestation (e.g. 4-hydroxyphenylacetic acid (HPAA), Fig.6.g.), others were found only in the extracts treated with a limited number of species. VA was rather removed by the fungi from the plant matrix, while GA, HT and CAi levels were generally more elevated when the material was treated with the plant-associated fungi.

The list of phenolic compounds includes HT, CA, along with an isomer (CAi), which might be a cis isomer of CA. Comparing Fig. 1. and Fig. 5. it can be seen, that HT and CA can be hydrolytic breakdown products of acteoside and related CPGs, like plantamajoside, lavandulifolioside, etc. (Ronsted et al., 2003) present in P. lanceolata. It is important to note, that spontaneous decomposition (i.e. in controls) to CA and HT was not observed (Fig. 6. a., c.). Other compounds, like HPAA, HBA, PCA and VA could be considered metabolites of the former phenolic compounds, via enzymatic oxydation of the side-chain and/or methylation. The breakdown of caffeic acid derivates to benzoic 
acid derivates by microbiological agents is described in the literature (Blum, 1998). As it can be seen, several fungal strains increase the amount of CA in the plant matrix (Fig. 6. a.), $(p<0.001)$. Especially incubation with strains 2, 5, 7 and 10 resulted in high amounts of caffeic acid, about three-fold increase was observed, leading to high concentrations of CA in solution.

Other detected metabolites were high amounts of organic acids secreted into the medium, but only by $A$. niger (see Fig. 4.): oxalic acid, citric acid, malonic acid, succinic acid, malic acid and fumaric acid were detected from the 9 day sample. Apart from these, monosaccharides, glycerol and fatty-acid TMS derivates were also detected - also from controls (Fig. 4.).

The above mentioned phenolic molecules all possess high antioxidant potential. Thus, it is likely, that fungal infestation can influence the antioxidant capacity of herbal materials. CA, being present in high concentration, can also alter the antimicrobial activity and microbiological pattern of the plant material in a real-world situation, as it was shown to have antimicrobial properties (Merkl et al., 2010). CA, VA, PCA, HT and HBA were all shown to have activity against bacteria (Escherichia coli, Bacillus cereus) and fungi (Fusarium culmorum) in the 1.25-10 mmol/1 range (Merkl et al., 2010).

It can be stated, that fungal infestation - in general - has led to accumulation of some phenolic acids and probably decomposition of other phenolic compounds, as well as acteoside in many cases. As the various fungal species were able to the produce the same array of phenolic compounds, most of the detected phenolic acids can be considered breakdown-products of the polyphenolic metabolites of plant origin, rather than being results of de novo synthesis.

The detected phenolic acids have different adsorption, pharmacokinetic profile and pharmacological effects compared to acteoside. Besides being powerful antioxidants that can be found in some functional food products (Bendini et al., 2007), several other possible uses were linked to these molecules, especially CA and its derivates. Caffeic acid and its phenylethyl ester (CAPE) are currently researched as possible anticancer agents - some metabolites of CA and CAPE were shown to inhibit 
cancer cell line development, via inhibition of glutathione-S-transferase (Kudugunti et al., 2011). Fungal transformation of the metabolite pattern of plant drugs therefore can yield additional effects under some circumstances.

\subsection{Effects of phenolic acids and model antioxidants on acteoside stability. During} incubation of the P. lanceolata water extract with different PAMs, some fungal strains significantly inhibited acteoside breakdown (see 2.2.). Concurrently, increase in concentrations of several phenolic acids were detected from extracts treated with these strains, as notable especially in the case of Eurotium species (see Fig. 2. b., c., and Fig. 6 - F2, F10).

The link between increased amount of several phenolic acids and acteoside protection was examined by comparing the residual acteoside concentration between the groups of fungi, which had different phenolic acid concentrations in the medium. As the concentrations of the phenolic acids was relatively correlated, measured values were subjected to principal component analysis after scaling and centering. Plotting the $9^{\text {th }}$ day samples in the plane of the two first principal components, it is visible, that the treatments form three distinct groups with respect to phenolic acid concentrations (Fig. 7.). The first two principal components (PC1, PC2) represent $79.98 \%$ of total variance of the variables. As it can be observed on Fig.7., distribution along PC1 is mainly influenced by concentrations of HBA, PCA, GA and CA, these parameters separate the two main clusters. It can be seen, that the first group (controls, $\boldsymbol{8}, \mathbf{9}, \boldsymbol{6})$ has significantly lower average amount of CA, GA, PCA and HBA $(p<0.01$, see Fig. 6. a, b, e, f; respectively) than the second group $(2,10,4,5,7) . A$. niger $(1)$ is an outlier from the above, mostly because of the high amount of HT detected in the sample incubated with it. A significant difference can be detected when comparing the residual acteoside amount between groups 1 and $2(p<$ 0.001). The residual amount of acteoside in the group with low phenolic acid content (controls, $8,9,6)$ was $17.62 \pm 24.35 \%$, while it was $79.75 \pm 7.77 \%$ in the group with high phenolic acid content $(2,10$, 
$4,5,7)$. Principal component regression analysis also showed, that the value of PC1 (mainly composed of concentrations of CA, GA, PCA and HBA) significantly influences residual acteoside concentration $(p<0.01)$. Therefore, the phenolic acid mixture was considered the agent responsible for the protection of acteoside in the current experiment. As the produced phenolic compounds are considered powerful antioxidants (Merkl et al., 2010), a mechanism involving prevention of oxidation was suspected behind the inhibition of degradation.

Because of the very poor solubility of the purified phenolic acids in the mildly acidic $P$. lanceolata water extract, model antioxidants were employes to test the effects of antioxidants on the stability of acteoside in the solution. Therefore, water extracts were spiked with different concentrations of sterile ascorbic acid (AA) and $\mathrm{Na}_{2}$ EDTA (EDTA) solutions, and samples were incubated for 15 days. Acteoside decomposition was quantified from samples, the results are plotted in Fig. 8. AA and EDTA significantly decreased the breakdown of acteoside $(p<0.001, p<0.001)$, both responses were concentration dependent. EDTA almost completely inhibited decomposition at 2.5 $\mathrm{mg} / \mathrm{ml}$ concentration, in particular, end-time concentration was significantly indifferent from the initial, Fig. 8. b. Based on these results, it seems likely, that acteoside is oxydatively decomposed during the incubation, and the antioxidants added can reduce this decomposition. This finding is in correspondence with the results of (Obied et al., 2005), who found that oxygen was essential for acteoside degradation in an olive mill waste model system, and phenolic compounds' recovery could be enhanced by addition of the antioxidant sodium metabisulphite. Antioxidants were also shown to inhibit decomposition of CA derivates in Echinacea (Chantal Bergeron, 2002).

Caffeic acid was shown to reduce the complexed Fe(III) by its dihydroxy-phenyl moiety (Hynes and O'Coinceanainn, 2004). Oxydation of caffeic acid results in diquinoic structures, the reaction is $\mathrm{pH}$ dependent. As acteoside has the same catechol group (Fig. 1.), it is likely that it can undergo the same reactions. While AA is a direct antioxidant, EDTA can rather be considered to exert indirect effects, the 
main antioxidant action attributed to EDTA is chelation of transition metals (e.g. $\mathrm{Cu}^{2+}$, iron, $\mathrm{Co}^{2+}$ ), which catalyse formation of reactive oxygen species. EDTA complexes of e.g. iron are more stable than complexes of polyphenolics, as shown for HT, PCA and CA by (Andjelković et al., 2006), and comparing the complex formation constants of acteoside and EDTA with iron, the same applies to acteoside as well (Zhao et al., 2005). Therefore, we think that acteoside was protected by EDTA by removing it from its metal complexes, thereby inhibiting its oxydation by transition metals.

The wide range of detected phenolic compounds in solution may protect acteoside by two mechanisms: by reducing the concentration of oxydants via reacting with them, and compete with actesoide in chelation of transition metals (especially those having a catechol group), thereby slowing down reactions between acteoside and transition metals. Indeed, many detected products have higher antioxidant (Vitamin C equivalent) values (VCEAC), than CA (e.g. PCA, VA), and others' VCEAC values are comparable (HPAA) to CA (Kim and Lee, 2004). Recall, that the concentrations of PCA, CA and HPAA these were major contributors to PC1, the value of which correlated with the inhibition of degradation of acteoside (Fig. 7.).

These results suggest, that the high amount of phenolic antioxidants present in the solution incubated with fungal strains may be the agents protecting acteoside from breakdown in the case of strains $2,4,5,7$ and $\mathbf{1 0}$. These antioxidants are likely to compete with acteoside in several reactions, resulting in preservation of more acteoside in solution. The acteoside protective effects may also be enhanced by uptake of transition metals for growth from the medium by the fungi, or chelation by siderophores (Saha et al., 2012). Intensive respiration leading to relative depletion of oxygen while the fungi are growing can also enhance this effect.

\section{Conclusions}


The PAMs isolated from the medicinal plant matrix were shown to have the ability to significantly alter the metabolite pattern of Plantago lanceolata extract. IG content decreased (aucubin, catalpol), while acteoside was decomposed by some strains, but was protected from spontaneous breakdown by other strains. The presence of high amounts of phenolic antioxidants was a common phenomenon of fungal infestation, and is likely to be result of the fungi metabolizing the plant's original polyphenolic substances. This pattern change caused by fungi might add new pharmaceutical effects to the plant material.

As fungal community is variable among production batches, the stability of chief constituents may not be predictable. Thus, it can be stated, that proper inhibition of the fungal activity in the production chain of these plant-based products is necessary. Fungi were found, that had the ability to selectively remove some of the metabolite mixture while not decomposing others. So, some of the nontoxic PAM could be used for medicinal plant matrix cleanup after further research and optimization, and proper selection of medicinal plant - PAM pairs might be a good starting point to develop interesting industrial applications and medicinal plant-based products.

Surprisingly, it was also shown that activity of fungal strains in the medicinal plants can also be beneficial for metabolite stability. Acteoside stability was significantly higher in solutions infested with the fungi producing high amounts of phenolic acids. With the help of transforming the concentrations of phenolic acids by principal component analysis, correlation was found between concentrations of phenolic acids and inhibition of acteoside decomposition. As acteoside could also be stabilized by addition of model antioxidants, we think that stabilization by fungal activity is likely to be the consequence of increasing concentrations of antioxidant phenolic acids in the water extract, produced by fungi.

Our paper contributes to the knowledge on interactions between microbiological agents and plant materials. Activity of fungi is typically described as undesired, because of the health risk 
associated with it in medicinal plant materials. In the current work, it was shown, that some of the plant-associated fungi can also act as beneficial agents - they can enrich the chemical pattern of the plant matrix, or increase the stability of bioactive constituents of plant origin.

\section{Experimental}

4.1. Chemicals. All chemicals (including solvents) were of analytical grade. For all purposes, bidistilled water was used. Aucubin, catalpol, caffeic acid were obtained from Sigma-Aldrich. Ascorbic acid and disodium EDTA were from Merck. Acteoside was purified from the dried leaves as described earlier (Gonda et al., 2012). Identity (differentiation from isoacteoside) was checked by ${ }^{1} \mathrm{H},{ }^{13} \mathrm{C}$ NMR studies on a Bruker Avance-II $500 \mathrm{MHz}$ spectrometer. Spectra were identical to those described in the literature earlier (Li et al., 2009).

4.2. Plant material. The leaves of Plantago lanceolata L. were collected from Hajdúsámson (Hungary) in August of 2007, and dried at temperatures not exceeding $50{ }^{\circ} \mathrm{C}$. The specimen are stored in the laboratory as Hs0-2007. The leaves were stored over adsorbent silica until further analysis.

4.3. Isolation and identification of plant-associated microorganisms. Ten strains of apparently different filamentous fungi were isolated and identified from the plant material. Complete characterization of the microbial flora was beyond the scope of this study. Isolation protocol was previously described (Gonda et al., 2012).

Identification consisted of examination of macroscopic and microscopic characteristics, DNA was sequenced from the isolated species. For DNA isolation, fungi were grown in modified minimalnitrate medium at $28^{\circ} \mathrm{C}, 300 \mathrm{rpm}$ for $48 \mathrm{~h}$ (Medium composition: $7.5 \mathrm{~g}$ disodium hydrogen phosphate dihydrate, $4.5 \mathrm{~g}$ potassium dihydrogen phosphate, $0.05 \mathrm{~g}$ of magnesium sulphate heptahydrate, $0.005 \mathrm{~g}$ 
of ferrous sulphate, $2.8 \mathrm{~g}$ of potassium chloride, $4 \mathrm{~g}$ of glucose and $3.5 \mathrm{~g}$ of potassium nitrate and $10 \mathrm{~g}$ mycological peptone to $1 \mathrm{~L}, \mathrm{pH}$ 6.8). Genomic DNA was isolated from the washed and lyophilized mycelia using the Genomic DNA Purification Kit (Fermentas) according to the instructions of the manufacturer. To amplify selected DNA sequences, the following primer pairs and annealing temperatures were used: ITS1, ITS4, (ITS-I; $55^{\circ} \mathrm{C}$ ) (White et al., 1990), Bt2a, Bt2b ( $\beta$-tubulin; $56.4^{\circ} \mathrm{C}$ ) (Glass and Donaldson, 1995), cmd5, cmd6 (calmodulin; $52.3^{\circ} \mathrm{C}$ ) (Hong et al., 2005) and EF1-728F, EF'-986R (translation elongation factor $1 \alpha ; 55^{\circ} \mathrm{C}$ ) (Carbone and Kohn, 1999). Primers were in part selected based on microscopic characteristics.

PCR assay $(50 \mu \mathrm{L})$ was carried out using $2 \mathrm{mM}$ magnesium chloride, $0.2 \mu \mathrm{M}$ primer (each), 0.2 mM dNTP, and 2 U Taq DNA polymerase (Fermentas) in a thermal cycler equipped with a heated lid. The steps of PCR reaction were as follows: (1) initial activation step, $95{ }^{\circ} \mathrm{C}, 5 \mathrm{~min}$; (2) DNA denaturation, $95{ }^{\circ} \mathrm{C}$, $45 \mathrm{sec}$; (3) annealing, $\mathrm{T} m-(2-5){ }^{\circ} \mathrm{C}$ (see above), $30 \mathrm{sec}$; (4) extension, $72{ }^{\circ} \mathrm{C}, 1$ $\min 20 \mathrm{sec}\left(35\right.$ cycles) and (5) final extension $10 \mathrm{~min}$ at $72^{\circ} \mathrm{C}$. PCR products were subjected to standard 1\% agarose gel-electrophoresis in TAE buffer (800 $\mu \mathrm{M}$ Tris, $400 \mu \mathrm{M}$ acetic acid, $20 \mu \mathrm{M} \mathrm{Na} 2^{-}$ EDTA; pH 8.5) and the purified bands (DNA Extraction Kit, Fermentas) were sequenced. Sequencing was performed using the ABI Big Dye Terminator 3.1 Kit on an automated DNA capillary sequencer (ABI PRISM 3100-Avant).

\subsection{Effects of plant-associated microorganisms on metabolite stability in P. lanceolata}

sterile water extracts. Direct sterilization of a plant material - with the metabolite pattern remaining intact - is very difficult to carry out. Therefore, to test the contribution of filamentous fungi to metabolite decomposition, a sterile water extract was incubated with isolated fungal strains. Besides the molecules of interest, water extracts also contain macromolecules and inorganic elements, which provides nutrients for the microbiological agents. 
Dried Plantago lanceolata leaves (100.0 g) were reduced to powder and subsequently extracted with $350 \mathrm{ml}$ of bidistilled water for 60 minutes with constant mixing at room temperature. The supernatant of the extract was centrifuged, filtered sterile and most of it was heated at $80{ }^{\circ} \mathrm{C}$ for 60 minutes to inactivate residual plant and microbial enzymes. Some solution was put aside as "raw" control solution.

The purified strains were spread on Saboraud's glucose agar (casein peptone $10 \mathrm{~g}$, meat peptone $10 \mathrm{~g}$, glucose monohydrate $40 \mathrm{~g}$ to $1 \mathrm{~L}, \mathrm{pH} 5.6 \pm 0.2$, solidified with $2 \%$ agar) supplemented with 50 $\mathrm{mg} / \mathrm{L}$ chloramphenicol, and allowed to grow at $28^{\circ} \mathrm{C}$ until a continuous layer was formed. One fourth of the mycelial surface was scraped off the medium surface, washed and suspended in $1000 \mu 1$ of sterile bidistilled water. Hundred microliters of this suspension was added to $10 \mathrm{ml}$ of the previously described substrate solution (water extract) and homogenized. The inoculated water extracts were divided to 250 $\mu 1$ aliquots, each was placed in a separate sterile test tube. The solutions were incubated in a sterile environment, with the tubes open, for 15 days. Samples were taken every three days. Each sampling day, samples were frozen at $-24^{\circ} \mathrm{C}$, liophilized and stored dry until measurements.

4.5. Determination of aucubin, catalpol and acteoside. Two-hundred fifty microlitres of $20 \%$ n-propanol was added to lyophilized samples, mixed, sonicated (3 minutes) and centrifuged at 18000 rpm to remove mycelia and precipitate. A 50 microliter part of the supernatant was evaporated to dryness and reuptaken in the same amount of water, the protocol was performed in triplicate. Thus, the procedure nullified the effect of different evaporation rates that were observed during the 15 days' incubation time. This procedure allowed higher recovery of compounds with less water solubility (e. g. acteoside and phenolic acids), and removal of the particles and mycelia was also much simpler to carry out (due to reduced viscosity).

The solutions were then analysed by capillary electrophoresis (CE). The method was a modified version of (Suomi et al., 2000). For quantification of iridoid glycosides and acteoside, a PrinCE-C 700 
capillary electrophoresis instrument with a diode array detector was used. Five-point standard calibration curves were used from both IGs and acteoside. IGs were detected at $200 \mathrm{~nm}$, acteoside at $350 \mathrm{~nm}$.

4.6. Identification and quantification of phenolic compounds. A $50 \mu 1$ aliquot of the redissolved (20\% n-propanol) liophilized sample was evaporated to dryness, and subsequently reuptaken in $50 \mu \mathrm{l}$ water, extracted with $1000 \mu \mathrm{l}$ of hexane, the hexane layer was discarded. The residual organic solvent was removed by vacuum centrifugation for 10 minutes in a Christ RVC 2-18 CD attached to a Christ CT02-50 solvent trap. Thereafter, the aliquot was extracted with $250 \mu 1$ ethyl acetate, $200 \mu \mathrm{l}$ of the ethyl acetate layer was evaporated to dryness, and reuptaken in $100 \mu 1$ acetonitrile with sonication (5 minutes). After complete dissolution, the sample was derivatized for GC-MS by adding $20 \mu \mathrm{l}$ of Supelco BSA + TMCS 5:1 Kit (Sylon BT) and incubated at room temperature for 10 minutes. One microliter of the sample was injected in 20:1 split mode to an Agilent 7890A GC 5975C MS instrument, with a HP-5 5\% Phenyl Methyl Siloxan HP-5 column (30 m x $320 \mu \mathrm{m}$ x $0.25 \mu \mathrm{m})$; flow rate was $1.5 \mathrm{ml} \mathrm{min}{ }^{-1}$ (helium). Front inlet was heated to $250{ }^{\circ} \mathrm{C}$. Initial oven temperature was 100 ${ }^{\circ} \mathrm{C}$, held for 1 minute; followed by a temperature gradient with rate of $15^{\circ} \mathrm{C} / \mathrm{min}$ for 15 minutes (up to $310^{\circ} \mathrm{C}$ ), finally, temperature was kept at $310^{\circ} \mathrm{C}$ until the end of the run (total length of 30 minutes). Transfer line temperature was set $320^{\circ} \mathrm{C}$, electron ionisation voltage used was $70 \mathrm{eV}$, ions were detected in the $\mathrm{m} / \mathrm{z}$ range 50-1000. For quantification of caffeic acid, five-point calibration curves were used, standards were prepared like samples, as described above.

4.7. Acteoside stability in water extracts with additional antioxidants. To test the effects of different antioxidants on the stability of acteoside, water extracts were spiked with sterile solutions ascorbic acid (final concentration 0.5 or $5 \mathrm{mg} / \mathrm{ml}$ ), or $\mathrm{Na}_{2} \mathrm{EDTA}$ (final concentration 0.25 or 2.5 $\mathrm{mg} / \mathrm{ml}$ ), and incubated for 15 days under the same conditions as described above (4.4.). Residual acteoside amount was quantified by $\mathrm{CE}$, as described above (4.5.). 
4.8. Principal component analysis and statistical analyses. For hypothesis testing, one-way ANOVA models were used, followed by Dunnett's test, where appropriate (overall level of significance was set at $p<0.05$ ). The $p$ values for hypotheses are given in sections Results and Discussion.

Differences were considered insignificant at $p>0.05$. The concentrations of the phenolic acids in the Plantago extracts after 9 days incubation with fungi (measurement: see 4.6.) were subjected to principal component analysis for dimensionality reduction. Principal component analysis and other calculations were done in R 2.14.1 (R Foundation for Statistical Computing, Vienna, Austria) statistical software, with package "multcomp" 1.2-12 (Hothorn et al., 2008), and "ggplot2" 0.9.1 (Wickham, 2009). 


\section{Acknowledgements}

This work has been supported by Hungarian National Research Foundation Grants OTKA K81370, F046493, GVOP-3.2.1.-2004-04-0110/3.0, GVOP-TST 3.3.1-05/1-2005-05-0004/3.0 and TÁMOP4.2.2-08/1/2008-2014, which is greatly acknowledged. The Bolyai J. research support for G. Vasas is acknowledged.

\section{References}

Andjelković, M., Van Camp, J., De Meulenaer, B., Depaemelaere, G., Socaciu, C., Verloo, M., Verhe, R., 2006. Iron-chelation properties of phenolic acids bearing catechol and galloyl groups. Food Chem. 98, 23-31.

Bendini, A., Cerretani, L., Carrasco-Pancorbo, A., Gómez-Caravaca, A.M., Segura-Carretero, A., Fernández-Gutiérrez, A., Lercker, G., 2007. Phenolic Molecules in Virgin Olive Oils: a Survey of Their Sensory Properties, Health Effects, Antioxidant Activity and Analytical Methods. An Overview of the Last Decade Alessandra. Molecules 12, 1679-1719.

Bensch, K., Groenewald, J.Z., Dijksterhuis, J., Starink-Willemse, M., Andersen, B., Summerell, B.A., Shin, H.-D., Dugan, F.M., Schroers, H.-J., Braun, U., Crous, P.W., 2010. Species and ecological diversity within the Cladosporium cladosporioides complex (Davidiellaceae, Capnodiales). Stud. Mycol. 67, 1-94.

Blum, U., 1998. Effects of microbial utilization of phenolic acids and their phenolic acid breakdown products on allelopathic interactions. J. Chem. Ecol. 24, 685-708.

Carbone, I., Kohn, L.M., 1999. A method for designing primer sets for speciation studies in filamentous ascomycetes. Mycologia 91, 553-556.

Chantal Bergeron, S.G., 2002. Stabilization of caffeic acid derivatives in Echinacea purpurea L. glycerin extract. J. Agric. Food Chem. 50, 3967-70.

Eder, M., Mehnert, W., 1998. Importance of concomitant compounds in plant extracts. Pharmazie 53, 285-293.

Egan, B., Hodgkins, C., Shepherd, R., Timotijevic, L., Raats, M., 2011. An overview of consumer attitudes and beliefs about plant food supplements. Food Funct. 2, 747-752.

Glass, N.L., Donaldson, G.C., 1995. Development of primer sets designed for use with the PCR to amplify conserved genes from filamentous ascomycetes. Appl. Env. Microbiol. 61, 1323.

Gonda, S., Toth, L., Gyemant, G., Braun, M., Emri, T., Vasas, G., 2012. Effect of High Relative Humidity on Dried Plantago lanceolata L. Leaves during Long-term Storage: Effects on Chemical Composition, Colour and Microbiological Quality. Phytochem. Anal. 23, 88-93.

Gutierrez, R.M.P., Gonzalez, A.M.N., Ramirez, A.M., 2012. Compounds Derived from Endophytes: A Review of Phytochemistry and Pharmacology. Curr. Med. Chem. 19, 2992-3030.

He, J., Hu, X., Zeng, Y., Li, Y., Wu, H., Qiu, R., Ma, W., Li, T., Li, C., He, Z., 2011. Advanced research 
on acteoside for chemistry and bioactivities. J. Asian Nat. Prod. Res. 13, 449-464.

Hong, S.B., Go, S.J., Shin, H.D., Frisvad, J.C., Samson, R.A., 2005. Polyphasic taxonomy of Aspergillus fumigatus and related species. Mycologia 97, 1316.

Hothorn, T., Bretz, F., Westfall, P., 2008. Simultaneous inference in general parametric models. Biom. J. 50, 346-363.

Hynes, M.J., O'Coinceanainn, M., 2004. The kinetics and mechanisms of reactions of iron(III) with caffeic acid, chlorogenic acid, sinapic acid, ferulic acid and naringin. J. Inorg. Biochem. 98, $1457-1464$.

Janda-Ulfig, K., Ulfig, K., Markowska, A., 2009. Extracellular Enzyme Profiles of Xerophilic Fungi Isolated from Dried Materials of Medicinal Plants. Polish J. Of Environ. Stud. 18, 391-397.

Kim, D.O., Lee, C.Y., 2004. Comprehensive study an vitamin C equivalent antioxidant capacity (VCEAC) of various polyphenolics in scavenging a free radical and its structural relationship. Crit. Rev. Food Sci. Nutr. 44, 253-273.

Kneifel, W., Czech, E., Kopp, B., 2002. Microbial contamination of medicinal plants--a review. Planta Med. 68, 5-15.

Kudugunti, S.K., Thorsheim, H., Yousef, M.S., Guan, L., Moridani, M.Y., 2011. The metabolic bioactivation of caffeic acid phenethyl ester (CAPE) mediated by tyrosinase selectively inhibits glutathione S-transferase. Chem. Biol. Interact. 192, 243-256.

Li, L.I., Liu, C., Chen, Z., Wang, J., Shi, D., Liu, Z., 2009. Isolation and Purification of Plantamajoside and Acteoside from Plant Extract of Plantago asiatica L. by High Performance Centrifugal Partition Chromatography. Chem Res Chinese U. 25, 817-821.

Lin, Z., Wen, J., Zhu, T., Fang, Y., Gu, Q., Zhu, W., 2008. Chrysogenamide a from an endophytic fungus associated with Cistanche deserticola and its neuroprotective effect on SH-SY5Y cells. J. Antibiot. 61, 81-85.

Liu, D., Li, X.-M., Meng, L., Li, C.-S., Gao, S.-S., Shang, Z., Proksch, P., Huang, C.-G., Wang, B.-G., 2011. Nigerapyrones A-H, alpha-Pyrone Derivatives from the Marine Mangrove-Derived Endophytic Fungus Aspergillus niger MA-132. J. Nat. Prod. 74, 1787-1791.

Meddah, N., Touhami, A.O., Douira, A., 2007. Hibiscus rosa-sinensis, a new host of Cochliobolus spicifer and Setosphaeria rostrata. Phytoprotection 88, 57-60.

Merk1, R., Hradkova, I., Filip, V., Smidrkal, J., 2010. Antimicrobial and Antioxidant Properties of Phenolic Acids Alkyl Esters. Czech. J. Food Sci. 28, 275-279.

Mukherjee, P.K., Kumar N, S., Heinrich, M., 2008. Plant made pharmaceuticals (PMPs) - Development of natural health products from bio-diversity. Indian J. Pharm. Educ. Res. 42, 113-121.

Obied, H.K., Allen, M.S., Bedgood, D.R., Jr, Prenzler, P.D., Robards, K., 2005. Investigation of Australian olive mill waste for recovery of biophenols. J. Agric. Food Chem. 53, 9911-9920.

Oyero, O., Oyefolu, A., 2009. Fungal contamination of crude herbal remedies as a possible source of mycotoxin exposure in man. Asian. Pac. J. Trop. Med. 2, 38-43.

Qiu, M., Xie, R., Shi, Y., Zhang, H., Chen, H., 2010. Isolation and identification of two flavonoidproducing endophytic fungi from Ginkgo biloba L. Ann. Microbiol. 60, 143-150.

Ronsted, N., Franzyk, H., Molgaard, P., Jaroszewski, J., Jensen, S., 2003. Chemotaxonomy and evolution of Plantago L. Plant Syst. Evol. 242, 63-82.

Roussel, S., Reboux, G., Dalphin, J., Pernet, D., Laplante, J., Millon, L., Piarroux, R., 2005. Farmer's lung disease and microbiological composition of hay: A case-control study. Mycopathologia 160, 273-279.

Saha, R., Saha, N., Donofrio, R.S., Bestervelt, L.L., 2012. Microbial siderophores: a mini review. J. Basic Microbiol. DOI: 10.1002/jobm.201100552

Senatore, F., Rigano, D., Formisano, C., Grassia, A., Basile, A., Sorbo, S., 2007. Phytogrowth- 
inhibitory and antibacterial activity of Verbascum sinuatum. Fitoterapia 78, 244-247.

Suomi, J., Siren, H., Hartonen, K., Riekkola, M., 2000. Extraction of iridoid glycosides and their determination by micellar electrokinetic capillary chromatography. J. Chromatogr. A 868, 7383.

Toth, B., Csosz, M., Dijksterhuis, J., Frisvad, J.C., Varga, J., 2007. Pithomyces chartarum as a pathogen of wheat. J. Plant Pathol. 89, 405-408.

Vertuani, S., Beghelli, E., Scalambra, E., Malisardi, G., Copetti, S., Dal Toso, R., Baldisserotto, A., Manfredini, S., 2011. Activity and stability studies of verbascoside, a novel antioxidant, in dermo-cosmetic and pharmaceutical topical formulations. Molecules 16, 7068-7080.

Wang, J.-M., Ding, G.-Z., Fang, L., Dai, J.-G., Yu, S.-S., Wang, Y.-H., Chen, X.-G., Ma, S.-G., Qu, J., Xu, S., Du, D., 2010. Thiodiketopiperazines Produced by the Endophytic Fungus Epicoccum nigrum. J. Nat. Prod. 73, 1240-1249.

White, T., Bruns, T., Lee, S., Taylor, J., 1990. Amplification and direct sequencing of fungal ribosomal RNA genes for phylogenetics, in: PCR Protocols: A Guide to Methods and Applications. Academic Press, pp. 322, 315.

Wickham, H., 2009. ggplot2: Elegant Graphics for Data Analysis, 2nd Printing. ed. Springer.

Zhao, C., Dodin, G., Yuan, C., Chen, H., Zheng, R., Jia, Z., Fan, B.-T., 2005. "In vitro" protection of DNA from Fenton reaction by plant polyphenol verbascoside. Biochim. Biophys. Acta General Subjects 1723, 114-123. 
Figure captions

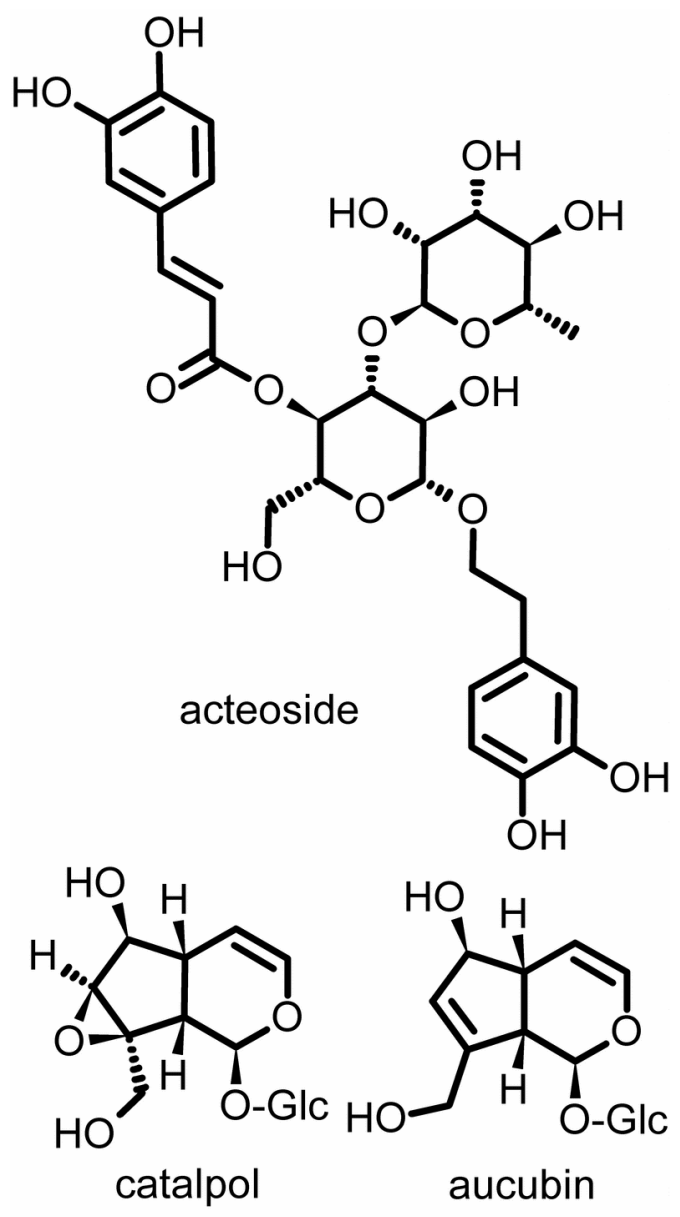

Fig. 1.

Chief bioactive metabolites of Plantago lanceolata leaves studied in the current paper - iridoid glycosides: aucubin and catalpol; caffeoyl phenylethanoid glycoside: acteoside. 

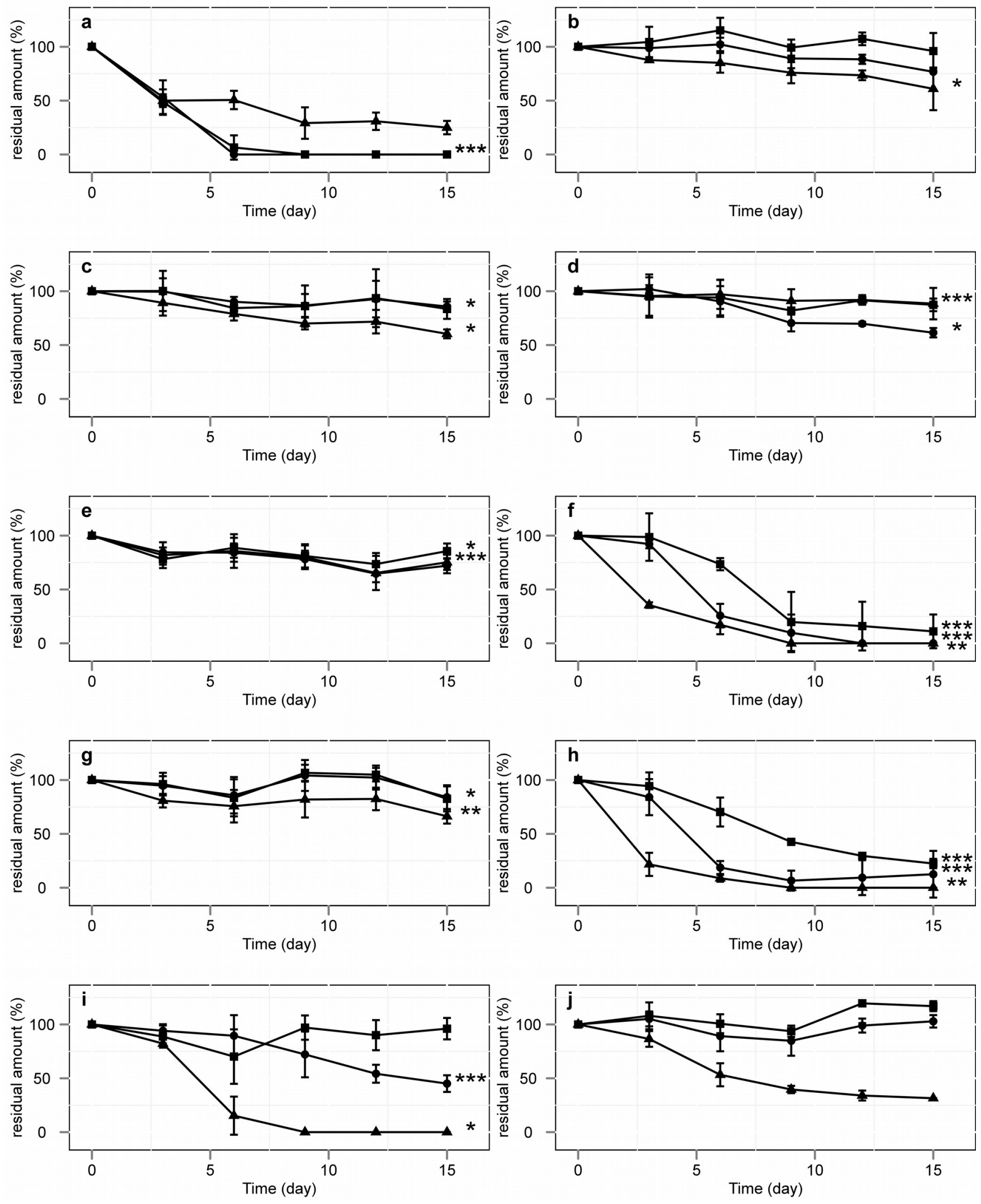
Fig. 2.

Changes in concentrations of bioactive metabolites in water extracts of Plantago lanceolata dried leaves infested with different plant-associated filamentous fungi, during 15 days of incubation.

Concentrations were measured with capillary electrophoresis, initial values are shown as $100 \%(n=3)$.

Significant differences for end-time values are shown against end-time values measured in controls (ANOVA followed by Dunnett-test, *,$p<0.05 ; * *, p<0.01 ; * * *, p<0.001$ ).

Subplots: a., 1, Aspergillus niger; b., 10, Eurotium repens; c., 2, Eurotium repens; d., 4, Aspergillus nidulans; e., 5, Eurotium amstelodami; f., 6, Cladosporium pseudocladosporioides; g., 7, Penicillium chrysogenum; h., 8, Bipolaris tetramera; i., 9, Epicoccum nigrum; j., control (inactivated, sterile). Legend: square, catalpol; circle, aucubin; triangle, acteoside. 


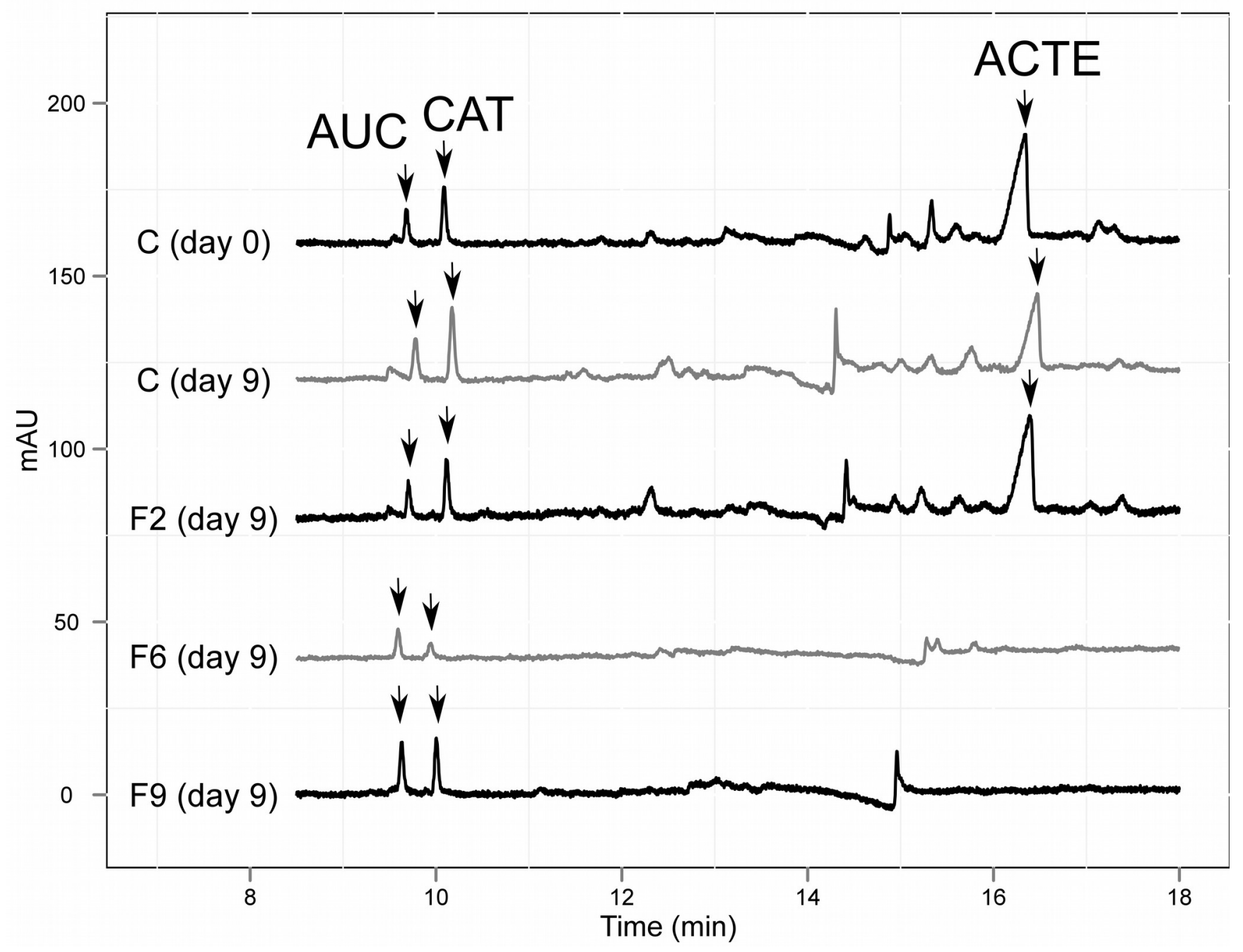

Fig. 3.

Selected electropherograms of Plantago lanceolata water extracts infested with different plantassociated filamentous fungi, after incubation for 9 days. Measurement was done at $200 \mathrm{~nm}$. Abbreviations: ACTE, acteoisde; AUC, aucubin; CAT, catalpol. Labels: F2, 2, Eurotium.repens; F6, 6, Cladosporium pseudocladosporioides; F9, 9, Epicoccum nigrum, C, control. 




Fig. 4.

Selected GC-MS total ion chromatograms of derivatized ethyl acetate fractions of Plantago lanceolata water extracts infested with different plant-associated filamentous fungi, after incubation for 9 days. Compounds detected as TMS derivates. Labels: $\mathrm{C}$, control at zero-time; $\mathrm{C} 9$, control at day 9; F1, 1 (Aspergillus niger) infested sample at day 9; F2, 2 (Eurotium repens) infested sample at day 9; F6, 6 (Cladosporium pseudocladosporioides) infested sample at day 9. Abbreviations: CA, caffeic acid, ; CAi, caffeic acid isomer; CiA, citric acid; GA, gentisic acid; H1, unidentified hexitol; H2, unidentified hexose; HBA: 4-hydroxy-benzoic acid; HPAA, 4-hydroxyphenylacetic acid; HT, hydroxy-tyrosol; PA, palmitic acid; PCA, protocatechuic acid; SA, stearic acid; VA, vanillic acid. 

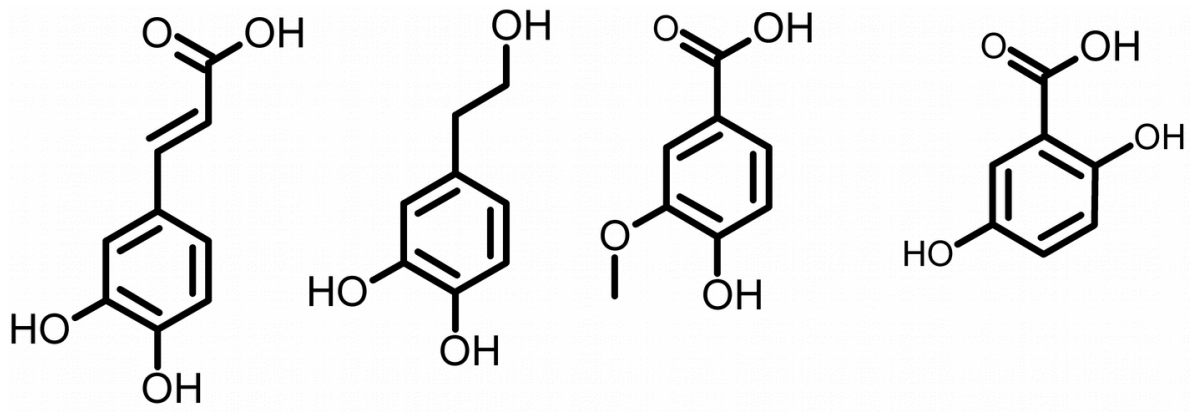

caffeic acid hydroxytyrosol vanillic acid gentisic acid<smiles>O=C(O)c1ccc(O)c(O)c1</smiles>

Protocatechuic acid<smiles>O=C(O)Cc1ccc(O)cc1</smiles>

p-hydroxy-phenylacetic acid<smiles>O=C(O)c1ccc(O)cc1</smiles>

p-hydroxy-benzoic acid

Fig. 5.

Selected chemical structures of compounds identified from the derivatized ethyl acetate fractions of Plantago lanceolata water extracts infested with different plant-associated filamentous fungi. 

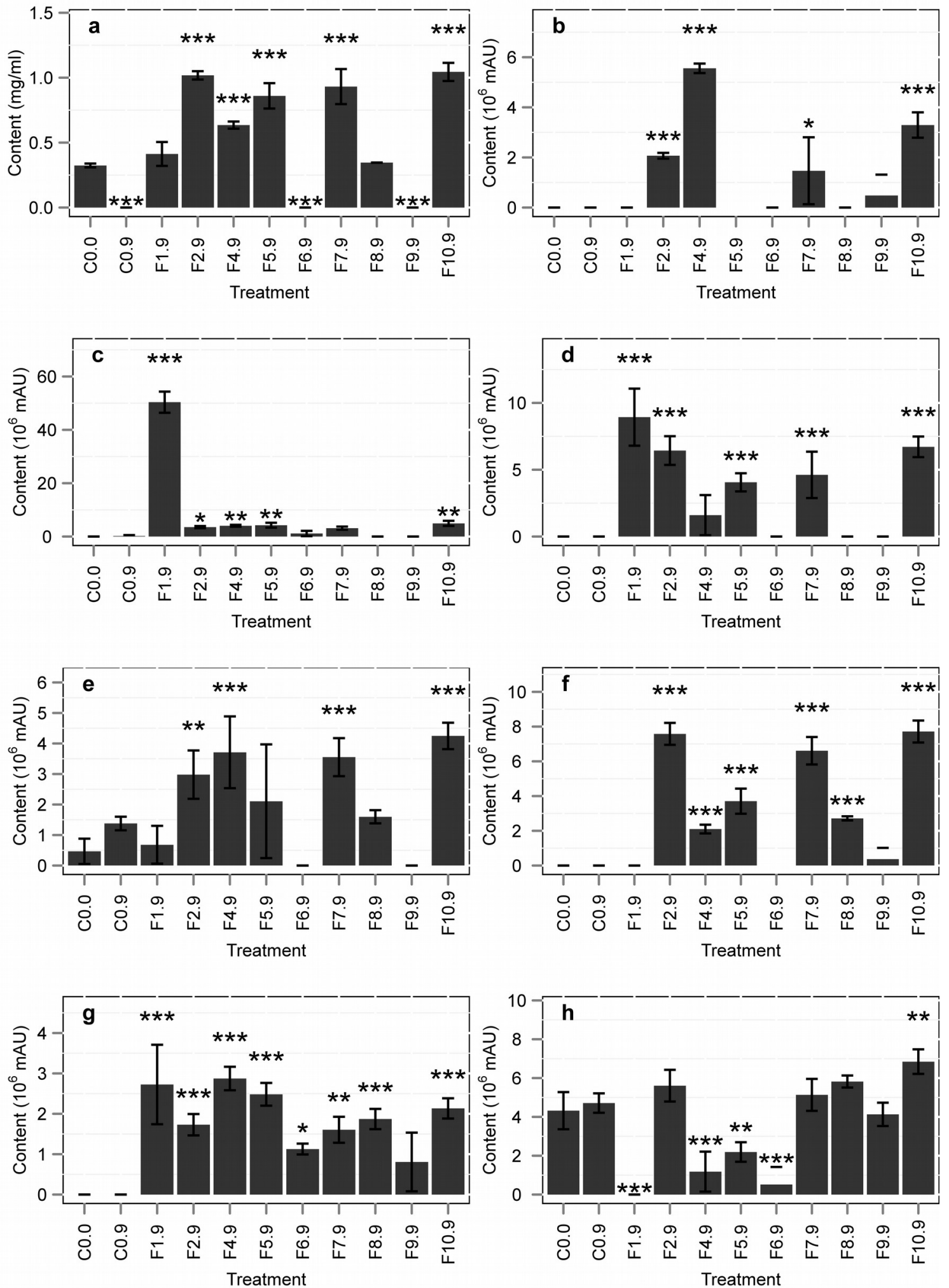

Fig. 6. 
Relative amount of selected GC-MS peaks in total ion chromatograms of derivatized ethyl acetate fractions of Plantago lanceolata water extracts infested with different plant-associated fungal strains isolated from the plant material, after incubation for 9 days. Compounds were detected as TMS derivates. Subplots: a., caffeic acid; b., gentisic acid; c., hydroxytyrosol; d., caffeic acid isomer; e., protocatechuic acid; f., 4-hydroxy-benzoic acid; g., 4-hydroxy-phenyl-acetic acid; h., vanillic acid. Abbreviations: mAU, milli-absorbance-unit. Significance: ANOVA followed by Dunnett-test, *, $p<0.05 ; * *, p<0.01 ; * * *, p<0.001$.

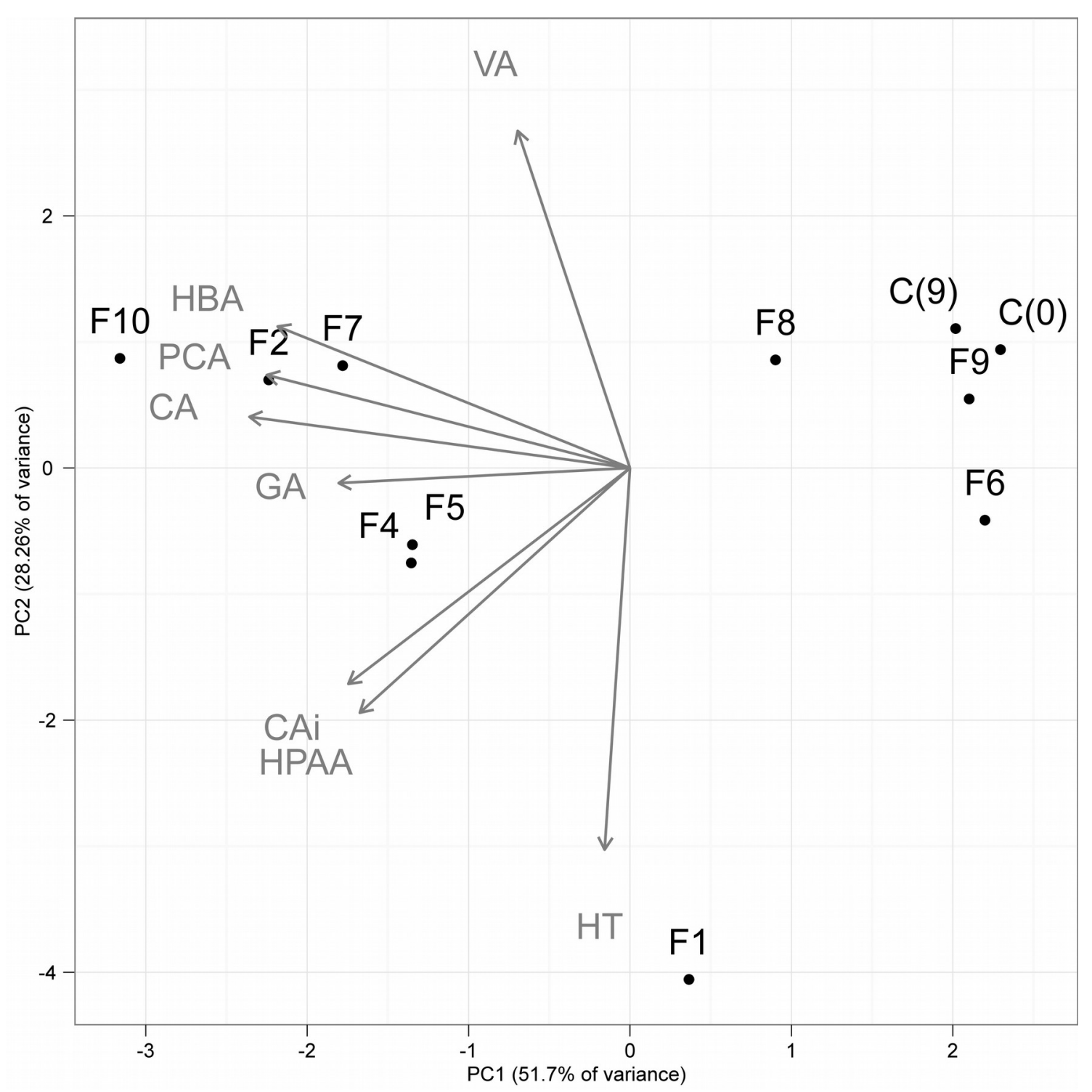




\section{Fig. 7.}

Two-dimensional visualization of phenolic acid concentration data for P. lanceolata extracts incubated with different fungal strains for 9 days. The dimensionality reduction by principal component analysis, the contribution of different variables to the first two principal component loadings are plotted as vectors (length adjusted for better visibility). Note that, the fungi are classified into three distinct groups. Legend: numbers, number of fungal strain (day 9); C0, control at zero time; C9, control at day 9. Abbreviations: CA, caffeic acid; CAi, caffeic acid isomer; GA, gentisic acid; HBA: 4-hydroxybenzoic acid; HPAA, 4-hydroxyphenylacetic acid; HT, hydroxy-tyrosol; PA, palmitic acid; PC, principal component; PCA, protocatechuic acid; VA, vanillic acid.


\section{Fig. 8.}

Effect of model antioxidants on stability of acteoside in sterile P. lanceolata water extracts incubated for 15 days. a., Effect of AA on stability of acteoside. Legend: square, Control; circle, 0.5 mg/ml AA; triangle, $5 \mathrm{mg} / \mathrm{ml} \mathrm{AA.} \mathrm{b.,} \mathrm{Effect} \mathrm{of} \mathrm{Na}_{2}$ EDTA on stability of acteoside. Legend: square, Control; circle, $0.25 \mathrm{mg} / \mathrm{ml}$ EDTA; triangle, $2.5 \mathrm{mg} / \mathrm{ml}$ EDTA. Significant differences for end-time values are shown 
against end-time values measured in controls (ANOVA followed by Dunnett-test, * $p<0.05$; **, $p<0.01 ; * * *, p<0.001)$. 


\section{Table 1.}

Details and results of identification of fungal strains isolated from the plant material. Accession numbers of uploaded sequences are shown.

List of abbreviations: $\beta \mathrm{T}$ : $\beta$-tubulin gene (partial); $\mathrm{Cm}$ : calmodulin sequence (partial); eF1: elongation factor 1 gene; ITS: internal transcribed spacer 1, 5.8S ribosomal RNA gene, and internal transcribed spacer 4 .

\begin{tabular}{|l|l|l|l|}
\hline $\begin{array}{l}\text { Strain } \\
\text { no. }\end{array}$ & Species identified & $\begin{array}{l}\text { Sequenced } \\
\text { gene }\end{array}$ & BankIt number \\
\hline 1 & Aspergillus niger & TS, $\beta$ T & JN862797, JN862798 \\
\hline 2 & Eurotium repens & ITS, Cm, $\beta$ T & $\begin{array}{l}\text { HM104685-HM104686, HM104687, } \\
\text { HM104688 }\end{array}$ \\
\hline 3 & Leptosphaerulina chartarum & ITS & JN862799 \\
\hline 4 & Aspergillus nidulans & ITS, $\beta$ T & - \\
\hline 5 & Eurotium amstelodami & ITS, Cm & JN862800, JN862801 \\
\hline 6 & $\begin{array}{l}\text { Cladosporium } \\
\text { pseudocladosporioides }\end{array}$ & eF1 & JN862802 \\
\hline 7 & Penicillium chrysogenum & $\beta T$ & JN862803 \\
\hline 8 & Bipolaris tetramera & ITS & JN862804 \\
\hline 9 & Epicoccum nigrum & ITS & JN862805 \\
\hline 10 & Eurotium repens & ITS, $\beta$ T & JN862806, JN862807 \\
\hline
\end{tabular}

